\title{
Multistep weighted essentially non-oscillatory scheme
}

\author{
Yiqing Shen ${ }^{* \dagger}$, Li Liu and Yan Yang \\ LHD, Institute of Mechanics, Chinese Academy of Sciences, Beijing 100190, China
}

\begin{abstract}
SUMMARY
A fifth-order accurate multistep weighted essentially non-oscillatory (WENO) scheme is constructed in this paper. Different from the traditional WENO schemes, which are designed to have $(2 r-1)$ th order accuracy in the smooth regions directly from $r$ candidate stencils, the new scheme is constructed through $(r-1)$ weighting steps. In each step, only two neighboring stencils are used to construct the intermediate fluxes (or the final flux), which are only one order higher than the fluxes obtained from the previous step. Henrick's mapping function is used in each step to satisfy the sufficient condition of fifth-order convergence for a fifth-order WENO scheme; hence, the new scheme is fifth-order accurate in smooth regions. The distinctive advantage of the new scheme is that it can improve the accuracy by one order higher than the traditional WENO schemes at transition points (connecting a smooth region and a discontinuity point); and hence, it improves the accuracy in the regions near discontinuities. Numerical examples show that the new scheme is robust and is less dissipative than the traditional fifth-order WENO schemes. Copyright @ 2014 John Wiley \& Sons, Ltd.
\end{abstract}

Received 10 October 2013; Revised 11 November 2013; Accepted 25 January 2014

KEY WORDS: numerical method; weighted essentially non-oscillatory scheme; shock wave; complex flowfield simulation

\section{INTRODUCTION}

The weighted essentially non-oscillatory (WENO) schemes have been developed and widely used in the past two decades. The basic idea of WENO scheme was firstly proposed by Liu et al. [1], in which the smoothest stencil of the ENO schemes [2] is replaced by a convex combination of the reconstructions on all candidate stencils. In order to obtain higher-order accuracy in smooth regions and keep the ENO property around discontinuities, the design of the weight of each stencil is very important.

Jiang and Shu [3] analyzed that an $r$ th order ENO scheme can only be converted into an $(r+$ 1)th order WENO scheme by using the smoothness indicator introduced by Liu et al. [1]. And then a classical fifth-order WENO scheme with a general framework for designing the smoothness indicators and weights was proposed by Jiang and Shu [3]. Henrick et al. [4] pointed out that the smoothness indicators of Jiang and Shu fail to improve the accuracy order of WENO scheme at critical points, where the first derivatives are zero. A mapping function is proposed by Henrick et al. [4] to obtain the optimal order at critical points. Recently, Borges et al. [5] suggested to use the whole five-points stencil to devise a smoothness indicator of higher order than the classical smoothness indicator proposed by Jiang and Shu [3] and developed the WENO-Z scheme. On the other hand, a class of WENO schemes higher than the fifth order are designed by Balsara and Shu in [6] and by Gerolymos et al. in [7]. Wang and Chen [8] proposed optimized WENO schemes for linear waves with discontinuities. Martin et al. [9] proposed a symmetric WENO method by means of a new

\footnotetext{
*Correspondence to: Yiqing Shen, LHD, Institute of Mechanics, Chinese Academy of Sciences, Beijing 100190, China.

†E-mail: yqshen@imech.ac.cn 
candidate stencil; the new schemes are $2 r$ th-order accurate and symmetric and less dissipative than Jiang and Shu's scheme.

Most of the aforementioned WENO schemes are designed to have $(2 r-1)$ th or $2 r$ th [9] order of accuracy in the smooth regions directly from $r$ th ENO schemes. Their focus is mainly on improving the accuracy in smooth regions, especially at the critical point $\left(f_{i}^{\prime}=0\right)$. Hence, for a solution containing discontinuities, these methods cannot obtain the optimal accuracy at transition points, which connect a smooth region and a discontinuity point. For example, Shen and Zha [10] analyzed the existing fifth-order WENO schemes and found that the accuracy of those schemes is of only third-order at transition points. This shortcoming can affect the general performance of the fifth-order WENO schemes, for example, the reduced accuracy in simulating the local separated flow induced by shock waves and the excessive numerical diffusion in the flows with shock/ turbulence interaction.

Because the solution at transition points is still smooth, ideally, the discretization accuracy of its first-order derivative can achieve fourth order if and only if a smooth stencil with five points is used. In other words, a fourth-order numerical flux can be constructed by using a smooth stencil with four points. In [10], Shen and Zha introduced two fourth-order fluxes combined with an estimation of smoothness/nonsmoothness of two adjacent four-point stencils to improve the accuracy. In early works [11], Shen et al. indicated that the smoothness indicator $I S_{k}$ of Jiang and Shu's WENO scheme does not satisfy the condition $\beta_{k}=D\left(1+O\left(\Delta x^{2}\right)\right)$ at the critical point $\left(f_{i}^{\prime}=0\right)$ and proposed a step-by-step reconstruction to avoid the strict condition. But the method does not satisfy the necessary and sufficient conditions for fifth-order convergence [4] at critical point.

In this paper, on the basis of the analysis [10], a new method for constructing WENO scheme is proposed. The idea of this method is to combine Henrick's mapping function with the idea of improving the accuracy of WENO-Z scheme one-by-one order. The distinctive advantage of the new constructing method is that it can improve the accuracy of WENO scheme near discontinuities without reducing the accuracy in smooth regions. Numerical examples show that the new scheme is less dissipative than the aforementioned fifth-order WENO schemes.

\section{THE NUMERICAL ALGORITHM}

For the hyperbolic conservation law in the form

$$
\frac{\partial u}{\partial t}+\frac{\partial f(u)}{\partial x}=0
$$

the flux function $f(u)$ can be split into two parts as $f(u)=f^{+}(u)+f^{-}(u)$ with $d f^{+}(u) / d u \geqslant 0$ and $d f^{-}(u) / d u \leqslant 0$. The semi-discretization form of (1) can be written as

$$
\frac{d u_{i}(t)}{d t}=-\frac{1}{\Delta x}\left(h_{i+1 / 2}-h_{i-1 / 2}\right)
$$

where the numerical flux is $h_{i+1 / 2}=h_{i+1 / 2}^{+}+h_{i+1 / 2}^{-}$. In this paper, only the positive part $h_{i+1 / 2}^{+}$ is described, and the superscript ' + ' is dropped for simplicity. The $h_{i+1 / 2}^{-}$is evaluated following the symmetric rule about $x_{i+1 / 2}$

\subsection{Weighted essentially non-oscillatory schemes [3-5]}

The flux of the fifth-order WENO scheme can be written as

$$
h_{i+1 / 2}=\sum_{k=0}^{2} \omega_{k} q_{k}
$$

where $q_{k}$ is the third-order flux on stencil $S_{k}^{3}=\left(x_{i+k-2}, x_{i+k-1}, x_{i+k}\right)$

$$
\left\{\begin{array}{l}
q_{0}=\frac{1}{3} f_{i-2}-\frac{7}{6} f_{i-1}+\frac{11}{6} f_{i} \\
q_{1}=-\frac{1}{6} f_{i-1}+\frac{5}{6} f_{i}+\frac{1}{3} f_{i+1} \\
q_{2}=\frac{1}{3} f_{i}+\frac{5}{6} f_{i+1}-\frac{1}{6} f_{i+2}
\end{array}\right.
$$


The weight $\omega_{k}$ is constructed as

$$
\omega_{k}=\frac{\alpha_{k}}{\alpha_{0}+\alpha_{1}+\alpha_{2}}
$$

with

$$
\alpha_{k}=\frac{c_{k}}{\left(\varepsilon+I S_{k}\right)^{p}}
$$

$I S_{k}$ is the smoothness indicator on stencil $S_{k}^{3}$. In [3], Jiang and Shu proposed $I S_{k}$ as

$$
I S_{k}=\sum_{l=1}^{2} \int_{x_{i-1 / 2}}^{x_{i+1 / 2}} \Delta x^{2 l-1}\left[q_{k}^{(l)}(x)\right]^{2} d x
$$

Taylor expansion of (6) gives

$$
\left\{\begin{array}{l}
I S_{0}=\frac{13}{12}\left(f_{i-2}-2 f_{i-1}+f_{i}\right)^{2}+\frac{1}{4}\left(f_{i-2}-4 f_{i-1}+3 f_{i}\right)^{2} \\
I S_{1}=\frac{13}{12}\left(f_{i-1}-2 f_{i}+f_{i+1}\right)^{2}+\frac{1}{4}\left(f_{i-1}-f_{i+1}\right)^{2} \\
I S_{2}=\frac{13}{12}\left(f_{i}-2 f_{i+1}+f_{i+2}\right)^{2}+\frac{1}{4}\left(3 f_{i}-4 f_{i+1}+f_{i+2}\right)^{2}
\end{array}\right.
$$

$c_{0}=0.3, c_{1}=0.6$, and $c_{2}=0.1$ are the optimal weights, which generate the fifth-order upstream central scheme. If $f_{i}^{\prime}=0$, Equation (7) gives $I S_{k}=D(1+O(\Delta x))$ and $\omega_{k}=c_{k}+O(\Delta x)$; this will degrade the convergence accuracy of the scheme $[4,5,11]$.

Henrick et al. [4] implemented a detailed truncation error analysis of Jiang and Shu's WENO scheme and gave the necessary and sufficient conditions for fifth-order convergence of WENO scheme as the following,

$$
\begin{gathered}
\sum_{k=0}^{2} A_{k}\left(\omega_{k}^{+}-\omega_{k}^{-}\right)=O\left(\Delta x^{3}\right), \\
\omega_{k}^{ \pm}-c_{k}=O\left(\Delta x^{2}\right) .
\end{gathered}
$$

where $A_{k}$ is the coefficient of third-order term $\left(\Delta x^{3}\right)$ in the Taylor series expansion of $q_{k}$ to the fifthorder upstream central approximation [4]. To satisfy the aforementioned conditions and improve the accuracy of weights $\omega_{k}$, a mapping function $g_{k}(\omega)$ is defined in [4] as

$$
g_{k}(\omega)=\frac{\omega\left(c_{k}+c_{k}^{2}-3 c_{k} \omega+\omega^{2}\right)}{c_{k}^{2}+\omega\left(1-2 c_{k}\right)},
$$

and an improved WENO scheme (WENO-M) is constructed by using $g_{k}(\omega)$ to generate new weights. WENO-M can obtain fifth-order convergence at critical points.

Borges et al. [5] proposed a sufficient condition for the fifth-order WENO scheme,

$$
\omega_{k}^{ \pm}-c_{k}=O\left(\Delta x^{3}\right)
$$

and introduced a parameter $\tau_{5}$ as

$$
\tau_{5}=\left|I S_{0}-I S_{2}\right|
$$

to construct a new smoothness indicator $I S_{k}^{z}$ as

$$
I S_{k}^{z}=\frac{I S_{k}+\varepsilon}{I S_{k}+\tau_{5}+\varepsilon}
$$

Using the $I S_{k}^{z}$ to construct the WENO scheme(called as WENO-Z), the new weights can satisfy the sufficient condition Equation (9) at critical points.

In all formulas, the parameter $\varepsilon$ is used to avoid the division by zero, $\varepsilon=10^{-6}$ is used in [3], and $\varepsilon=10^{-40}$ is used in $[4,5]$. $p$ is chosen to increase the difference of scales of distinct weights at nonsmooth parts of the solution. 


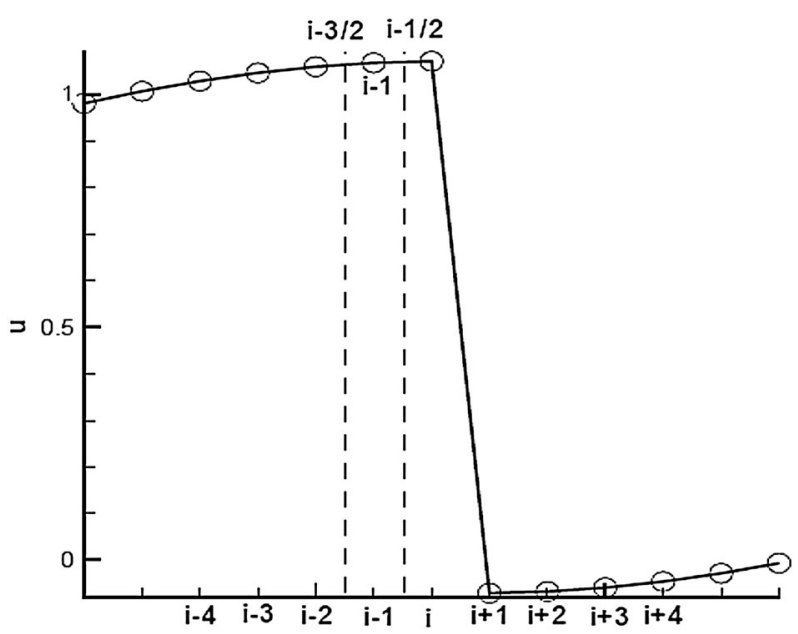

Figure 1. The sketch of transition point.

\subsection{Accuracy analysis at transition point [10]}

The analysis of fifth-order WENO schemes of Shen and Zha [10] shows that the accuracy of fifthorder WENO schemes is reduced at the transition point from smooth region to discontinuous point and vice versa. For completeness, the analysis is briefly introduced here. Figure 1 is taken as an example.

Points $i$ and $i+1$ are two discontinuity points. Point $i-1$ is called transition point in this paper. At point $i-1$, the stencil $S_{(i-1)-1 / 2}^{5}=\left(x_{i-4}, x_{i-3}, \cdots, x_{i}\right)$ is a smooth stencil; hence, $h_{(i-1)-1 / 2}$ obtained by the process of WENO-Z or WENO-JS scheme is a fifth-order flux. However, for stencil $S_{(i-1)+1 / 2}^{5}=\left(x_{i-3}, x_{i-2}, \cdots, x_{i+1}\right)$, there is a discontinuity at stencil $S_{2}^{3}=\left(x_{i-1}, x_{i}, x_{i+1}\right)$, so

$$
I S_{2}>>I S_{0}, I S_{1}
$$

no matter whether WENO-Z or WENO-JS is used. For calculating the flux $h_{(i-1)+1 / 2}$, it is easy to find

$$
\omega_{0} \rightarrow \frac{1}{7}, \omega_{1} \rightarrow \frac{6}{7}, \omega_{2} \rightarrow 0
$$

Under the cases of $\Delta x \rightarrow 0$, there are

$$
h_{(i-1)-1 / 2}=\frac{1}{30} f_{i-4}-\frac{13}{60} f_{i-3}+\frac{46}{60} f_{i-2}+\frac{9}{20} f_{i-1}-\frac{1}{20} f_{i}
$$

and

$$
h_{(i-1)+1 / 2}=\frac{1}{20} f_{i-3}-\frac{13}{42} f_{i-2}+\frac{41}{42} f_{i-1}+\frac{2}{7} f_{i}
$$

Applying Taylor series expansion, there is

$$
\frac{1}{\Delta x}\left(h_{(i-1)+1 / 2}-h_{(i-1)-1 / 2}\right)=f_{i-1}^{\prime}+O\left(\Delta x^{3}\right)
$$

That is, at the transition point $i-1$, the fifth-order WENO scheme only gives third-order accuracy.

Similarly, it can be proven that the $(2 r-1)$ th-order WENO scheme only gives $r$ th-order accuracy at points $i-r+2, i-r+3, \cdots, i-1$. For example, the seventh-order WENO scheme only gives fourth-order accuracy at points $i-2, i-1$.

Because the solution at transition point is still smooth, ideally, the discretization accuracy of its first-order derivative can achieve fourth order if and only if a smooth stencil with five points is used. In other words, a fourth-order numerical flux can be constructed by using a smooth stencil with four points. 
To improve the accuracy at transition point, in [10], a new method is constructed as

$$
h_{i+1 / 2}=\left\{\begin{array}{l}
q_{0}^{4}, \text { if } \tau_{4}^{0} \leqslant \min \left(I S_{k}\right) \text { and } \tau_{4}^{1} \geqslant \min \left(I S_{k}\right) \\
q_{1}^{4}, \text { if } \tau_{4}^{0} \geqslant \min \left(I S_{k}\right) \text { and } \tau_{4}^{1} \leqslant \min \left(I S_{k}\right) \\
h^{W E N O-Z}, \text { otherwise }
\end{array}\right.
$$

where

$$
\left\{\begin{array}{l}
q_{0}^{4}=\frac{1}{12}\left(f_{i-2}-5 f_{i-1}+13 f_{i}+3 f_{i+1}\right) \\
q_{1}^{4}=\frac{1}{12}\left(-f_{i-1}+7 f_{i}+7 f_{i+1}-f_{i+2}\right)
\end{array}\right.
$$

are two fourth-order fluxes, which can be written as the combination of the third-order fluxes $q_{k}$ in Equation (4) as the following,

$$
\left\{\begin{array}{l}
q_{0}^{4}=c_{0}^{4,0} q_{0}+c_{1}^{4,0} q_{1} \\
q_{1}^{4}=c_{0}^{4,1} q_{1}+c_{1}^{4,1} q_{2}
\end{array}\right.
$$

The constant coefficients $c_{k}^{4, l}(k=0,1 ; l=0,1)$ are determined as

$$
c_{0}^{4,0}=0.25, \quad c_{1}^{4,0}=0.75 ; \quad c_{0}^{4,1}=0.5, c_{1}^{4,1}=0.5 .
$$

$\tau_{4}^{0}$ and $\tau_{4}^{1}$ are defined as $\tau_{4}^{0}=\left|I S_{0}-I S_{1}\right|$ and $\tau_{4}^{1}=\left|I S_{1}-I S_{2}\right|$, respectively.

Although the estimation of a transition point introduced in (12) is not so accurate, the scheme (12) is effective to improve the accuracy at transition point and keep the ENO property [10].

\subsection{Multistep weighting method for WENO scheme}

Different from the aforementioned method (12), in this paper, a new constructing method for WENO scheme is proposed by combining Henrick's mapping function [4] and the idea of improving the accuracy of WENO-Z scheme one-by-one order [11]. Figure 2 can be used to illustrate the method.

In the first step, two fourth-order weighted fluxes are constructed as the following,

$$
\left\{\begin{array}{l}
h_{0}^{4}=\omega_{0}^{4,0} q_{0}+\omega_{1}^{4,0} q_{1} \\
h_{1}^{4}=\omega_{0}^{4,1} q_{1}+\omega_{1}^{4,1} q_{1}
\end{array}\right.
$$

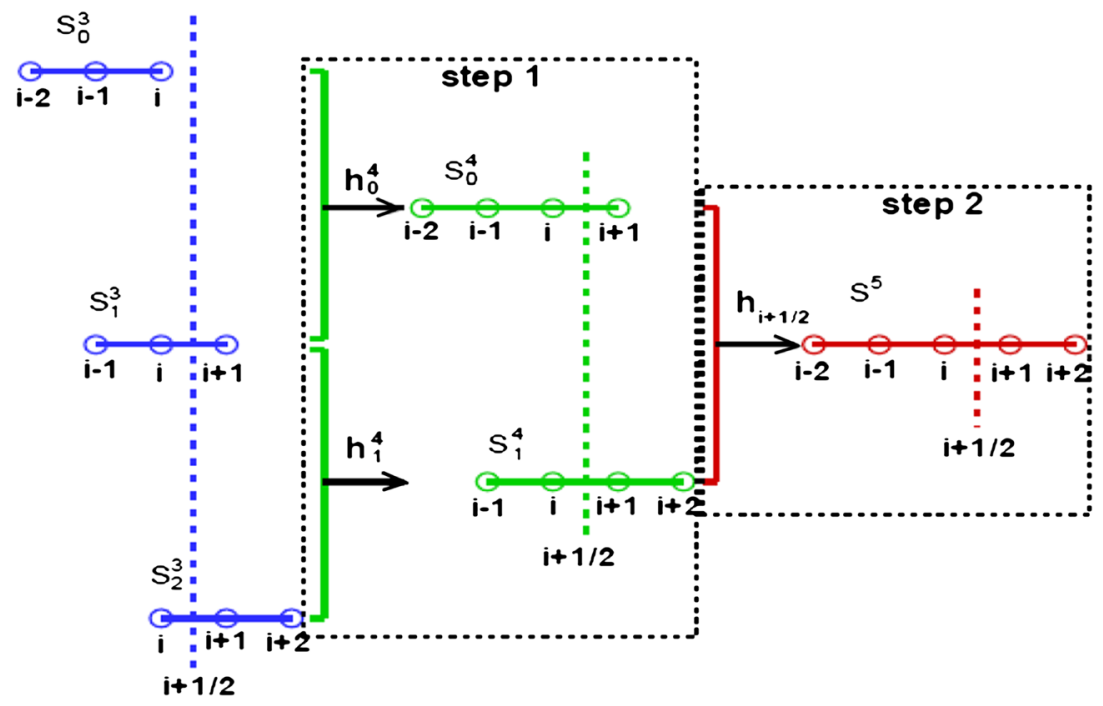

Figure 2. Multistep constructing process. 
The weights $\omega_{k}^{4, l}(k=0,1 ; l=0,1)$ are calculated by combining the method of WENO-Z scheme and mapping function,

$$
\omega_{k}^{4, l}=\frac{g_{k}\left(\psi_{k}^{4, l}\right)}{\sum_{k} g_{k}\left(\psi_{k}^{4, l}\right)}, \quad \psi_{k}^{4, l}=\frac{\alpha_{k}^{4, l}}{\sum_{k} \alpha_{k}^{4, l}}, \quad \alpha_{k}^{4, l}=c_{k}^{4, l}\left(1+\frac{\tau_{4}^{l}}{I S_{l+k}+\varepsilon}\right), \quad \tau_{4}^{l}=\left|I S_{l+1}-I S_{l}\right| .
$$

The optimal weights $c_{k}^{4, l}(k=0,1 ; l=0,1)$ are the same as in Equation (12).

In the second step, the final fifth-order weighted flux is obtained as the following

$$
h_{i+1 / 2}=\omega_{0} h_{0}^{4}+\omega_{1} h_{1}^{4}
$$

where $\omega_{k}=\frac{g_{k}\left(\psi_{k}\right)}{\sum_{k} g_{k}\left(\psi_{k}\right)}, \quad \psi_{k}=\frac{\alpha_{k}}{\sum_{k} \alpha_{k}}, \quad \alpha_{k}=c_{k}^{5}\left(1+\frac{\tau_{5}}{I S_{2 k}+\varepsilon}\right), \quad \tau_{5}=\left|I S_{2}-I S_{0}\right| . c_{0}^{5}=0.4$ and $c_{1}^{5}=0.6$ are the optimal weights, which make the linear combination of the two fourth-order linear fluxes $\left(q_{0}^{4}\right.$ and $q_{1}^{4}$ in Equation (12)) to the fifth-order upstream central flux.

Now, let us analyze the accuracy of the new methods (13) and (14). Using Taylor expansion, there is

$$
\tau_{4}^{l}=\left|f_{i}^{\prime} f_{i}^{\prime \prime \prime}\right| \Delta x^{4}+O\left(\Delta x^{5}\right)
$$

so there is

$$
\psi_{k}^{4, l}=\left\{\begin{array}{l}
c_{k}^{4, l}+O\left(\Delta x^{2}\right), \quad \text { if } \quad f_{i}^{\prime} \neq 0 \\
c_{k}^{4, l}+O(\Delta x), \quad \text { if } \quad f_{i}^{\prime}=0 \text { and } f_{i}^{\prime \prime} \neq 0
\end{array}\right.
$$

Applying the property of mapping function $g_{k}(\omega)$

$$
g_{k}\left(\psi_{k}^{4, l}\right)=c_{k}^{4, l}+\frac{\left(\psi_{k}^{4, l}-c_{k}^{4, l}\right)^{3}}{c_{k}^{4, l}-\left(c_{k}^{4, l}\right)^{3}}+\cdots,
$$

it is easy to find that

$$
\omega_{k}^{4, l}= \begin{cases}c_{k}^{4, l}+O\left(\Delta x^{6}\right), & \text { if } \quad f_{i}^{\prime} \neq 0 \\ c_{k}^{4, l}+O\left(\Delta x^{3}\right), & \text { if } \quad f_{i}^{\prime}=0 \text { and } f_{i}^{\prime \prime} \neq 0\end{cases}
$$

Similarly, there is

$$
\omega_{k}= \begin{cases}c_{k}^{5}+O\left(\Delta x^{9}\right), & \text { if } \quad f_{i}^{\prime} \neq 0 \\ c_{k}^{5}+O\left(\Delta x^{3}\right), & \text { if } \quad f_{i}^{\prime}=0 \text { and } f_{i}^{\prime \prime} \neq 0\end{cases}
$$

That means, in the aforementioned two steps, the sufficient condition of (9) is always satisfied no matter whether $f_{i}^{\prime} \neq 0$ or $f_{i}^{\prime}=0$ and $f_{i}^{\prime \prime} \neq 0$. Hence, there is no accuracy reducing in the multistep process; the method of (13) and (14) is fifth-order accurate in smooth regions.

If $x_{i}$ is a transition point (for example, the discontinuity is between $x_{i+1}$ and $x_{i+2}$ ), then in the first step, the fourth-order flux $h_{0}^{4}$ is obtained from Equation (13). In the second step, the final flux $h_{i+1 / 2}$ is approximated as $h_{i+1 / 2} \rightarrow h_{0}^{4}$ because of $I S_{2}>>I S_{0}$ and $\omega_{1} \rightarrow 0$ in Equation (14). Hence, the fourth-order accuracy at the transition point is obtained.

It should be noted that the calculation of (13) and (14) is more expensive than WENO-M and WENO-Z schemes because six weights are calculated by using the techniques of both WENO-M and WENO-Z schemes. However, because the multistep processes are only necessary near discontinuities, some efficient methods can be used to reduce the expensive calculation. For example, using a shock-detecting method to detect nonsmooth stencil, a hybrid scheme can be constructed as following,

$$
h_{i+1 / 2}= \begin{cases}h_{i+1 / 2}^{\text {present }}, & \text { if stencil } S_{i}^{5} \text { is a non-smooth stencil } \\ h_{i+1 / 2}^{\text {other }}, & \text { otherwise }\end{cases}
$$


Table I. Comparison of accuracy, $\mathrm{T}=2$.

\begin{tabular}{lrcclc}
\hline Scheme & $\mathrm{N}$ & $L_{1}$ error & $L_{1}$ order & \multicolumn{1}{c}{$L_{\infty}$ error } & $L_{\infty}$ order \\
\hline WENO-Z & 40 & $0.217102 \mathrm{e}-3$ & - & $0.677211 \mathrm{e}-4$ & - \\
& 80 & $0.649393 \mathrm{e}-5$ & 5.063 & $0.237405 \mathrm{e}-5$ & 4.834 \\
& 160 & $0.204882 \mathrm{e}-6$ & 4.986 & $0.785200 \mathrm{e}-7$ & 4.918 \\
& 320 & $0.748874 \mathrm{e}-8$ & 4.774 & $0.250232 \mathrm{e}-8$ & 4.971 \\
& 640 & $0.364893 \mathrm{e}-9$ & 4.359 & $0.779779 \mathrm{e}-10$ & 5.004 \\
WENO-M & 40 & $0.210766 \mathrm{e}-3$ & - & $0.672781 \mathrm{e}-4$ & - \\
& 80 & $0.648426 \mathrm{e}-5$ & 5.023 & $0.225867 \mathrm{e}-5$ & 4.897 \\
& 160 & $0.204671 \mathrm{e}-6$ & 4.986 & $0.720345 \mathrm{e}-7$ & 4.971 \\
& 320 & $0.640983 \mathrm{e}-8$ & 4.997 & $0.226830 \mathrm{e}-8$ & 4.989 \\
& 640 & $0.200631 \mathrm{e}-9$ & 4.998 & $0.710974 \mathrm{e}-10$ & 4.996 \\
& 40 & $0.203332 \mathrm{e}-3$ & - & $0.714827 \mathrm{e}-4$ & - \\
& 80 & $0.649369 \mathrm{e}-5$ & 4.969 & $0.229242 \mathrm{e}-5$ & 4.963 \\
& 160 & $0.204635 \mathrm{e}-6$ & 4.988 & $0.724031 \mathrm{e}-7$ & 4.985 \\
& 320 & $0.640982 \mathrm{e}-8$ & 4.997 & $0.227140 \mathrm{e}-8$ & 4.994 \\
& 640 & $0.200642 \mathrm{e}-9$ & 4.998 & $0.711126 \mathrm{e}-10$ & 4.997 \\
\hline
\end{tabular}

where, $h_{i+1 / 2}^{\text {present }}$ denotes the present multistep WENO scheme, $h_{i+1 / 2}^{\text {other }}$ can be other schemes constructed within the stencil $S_{i}^{5}$, for example, different WENO schemes, upstream central scheme, and compact schemes. In [12], Shen and Zha proposed a parameter-free shock-detecting method, that is, if $\left|I S_{2}-I S_{0}\right|>\min \left(I S_{k}\right)$, then $S_{i}^{5}$ is regarded as a nonsmooth stencil. This shock-detecting method is verified to be robust and effective [12]. Following the idea of Equation (17), it is promising to develop various high efficiency and high-order low diffusion schemes.

\section{NUMERICAL EXAMPLES}

In this paper, the fourth-order Runge-Kutta-type method [13] is used for the time integration.

\subsection{Linear transport equation}

The linear transport problems are controlled by

$$
\left\{\begin{array}{l}
\frac{\partial u}{\partial t}+\frac{\partial u}{\partial x}=0, \quad-1 \leqslant x \leqslant 1 \\
u(x, 0)=u_{0}(x), \quad \text { periodic boundary }
\end{array}\right.
$$

1. Initial solution $u_{0}(x)=\sin \left(\pi x-\frac{\sin (\pi x)}{\pi}\right)$

This solution has two critical points. Table I gives the errors and accuracy order. It can be seen that, for the smooth solution, the present scheme obtains the same fifth-order accuracy as WENO-M and WENO-Z schemes.

2. Initial solution

$$
u_{0}(x)=\left\{\begin{array}{l}
-\sin (\pi x)-\frac{1}{2} x^{3}, \quad-1<x \leqslant 0 \\
-\sin (\pi x)-\frac{1}{2} x^{3}+1,0<x \leqslant 1
\end{array}\right.
$$

Figure 3(a) shows the numerical solutions with $\mathrm{N}=200$ at $t=6$. It can be seen that, near the discontinuity, the present method obtains more accurate solution than WENO-Z and WENO$\mathrm{M}$ schemes. Figure 3(b) provides the comparison of the present scheme and the seventh-order WENO scheme (WENO-7) of Balsara and Shu [6]. As shown in this figure, the present scheme is even more accurate than WENO-7 scheme near discontinuity. This agrees with the analysis in Section 2.2, that is, at transition point $i-1$ (in the sketch Figure 1), both schemes are fourthorder accurate, but at the point $i-2$, WENO-7 scheme is only fourth-order accurate, whereas the present scheme is fifth-order accurate. 

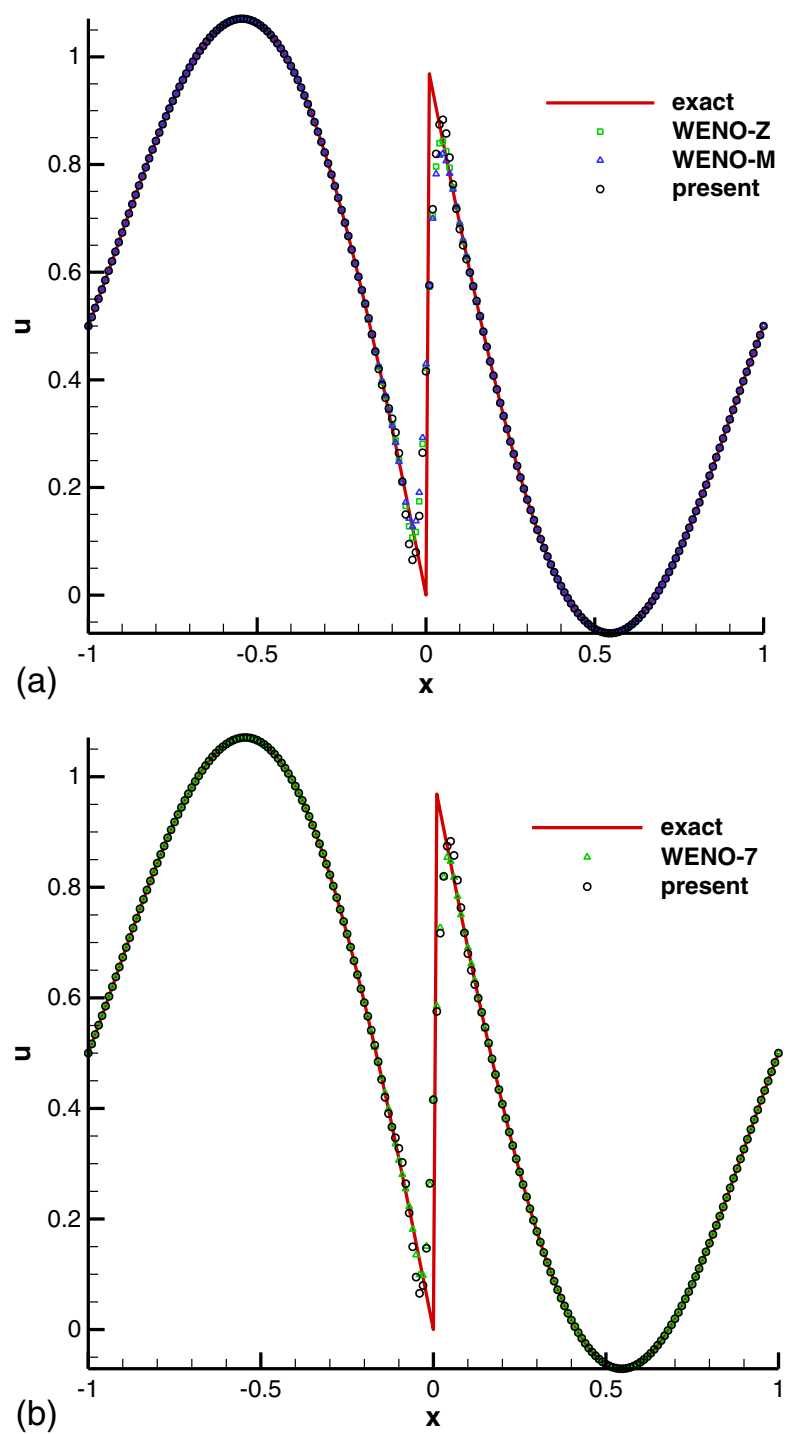

Figure 3. Numerical results with the initial solution Equation (19), $t=6$.

3. Initial solution

$$
u_{0}(x)= \begin{cases}\frac{1}{6}(G(x, \beta, z-\delta)+G(x, \beta, z+\delta)+4 G(x, \beta, z)) & -0.8 \leqslant x \leqslant-0.6 \\ 1, & -0.4 \leqslant x \leqslant-0.2 \\ 1-|10(x-0.1)|, & 0 \leqslant x \leqslant 0.2 \\ \frac{1}{6}(F(x, \alpha, a-\delta)+F(x, \alpha, a+\delta)+4 F(x, \alpha, a)) & 0.4 \leqslant x \leqslant 0.6 \\ 0, & \text { otherwise }\end{cases}
$$

where

$$
G(x, \beta, z)=e^{-\beta(x-z)^{2}}, \quad F(x, \alpha, a)=\sqrt{\max \left(1-\alpha^{2}\left(x-a^{2}\right), 0\right)}
$$

the constants are taken as $a=0.5 z=-0.7, \delta=0.005$, and $\beta=\log 2 / 36 \delta^{2}$. The solution contains a smooth combination of Gaussians, a square wave, a sharp triangle wave, and a half ellipse. Figure 4(a) and (b) gives the comparison of the results of the fifth-order WENO-Z scheme, WENO-M scheme, and the present method. The grid number is $\mathrm{N}=200$. It can be seen that the new method improves the accuracy near discontinuities as well as at critical points. The comparison of the present scheme and WENO-7 scheme is also given in Figure 5(a) and (b). 

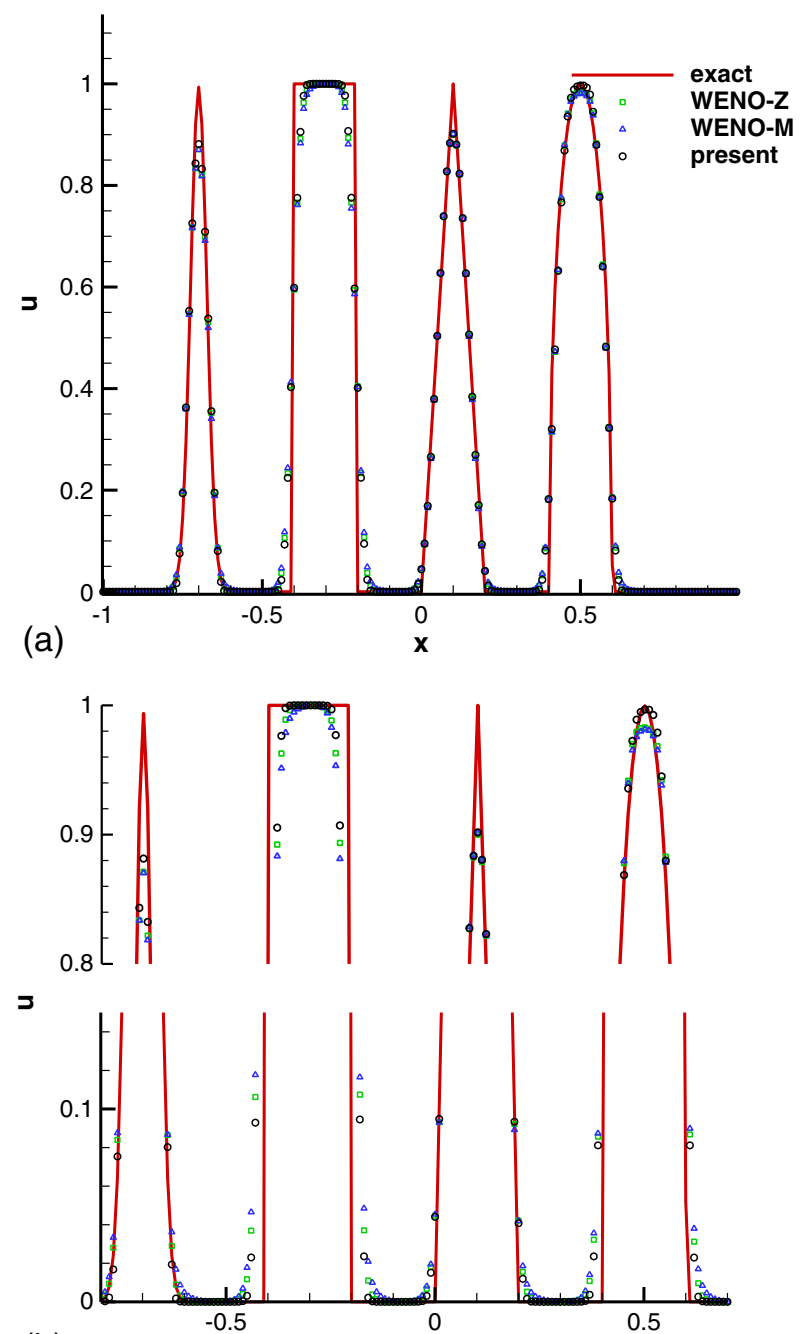

(b)

$\mathbf{x}$

Figure 4. (a) Numerical results with the initial solution Equation (20), $t=6$. (b) Locally enlarged plot of Figure 4(a).

Same as in the previous case, for the regions containing discontinuities as well as the ellipse wave, the present scheme obtains more accurate solution than WENO-7 scheme.

\subsection{Nonlinear transport equation}

The nonlinear transport equation

$$
\frac{\partial u}{\partial t}+u \frac{\partial u}{\partial x}=0, \quad 0 \leqslant x \leqslant 2 \pi
$$

is solved with initial and boundary conditions:

$$
u_{0}(x)=0.3+0.7 \sin (x) 0 \leqslant x \leqslant 2 \pi, \text { periodic boundary. }
$$

The flux splitting $f^{ \pm}=(f \pm a u) / 2$ is applied, where $f=u^{2} / 2$ and $a=\max \left(u_{i}\right)$. Figure 6 shows the results at $t=2$ with grid number of $\mathrm{N}=80$. It can be seen that, near the shock, the solution calculated by the present scheme is closer to the discontinuous solution than WENO-Z and WENO-M schemes. 

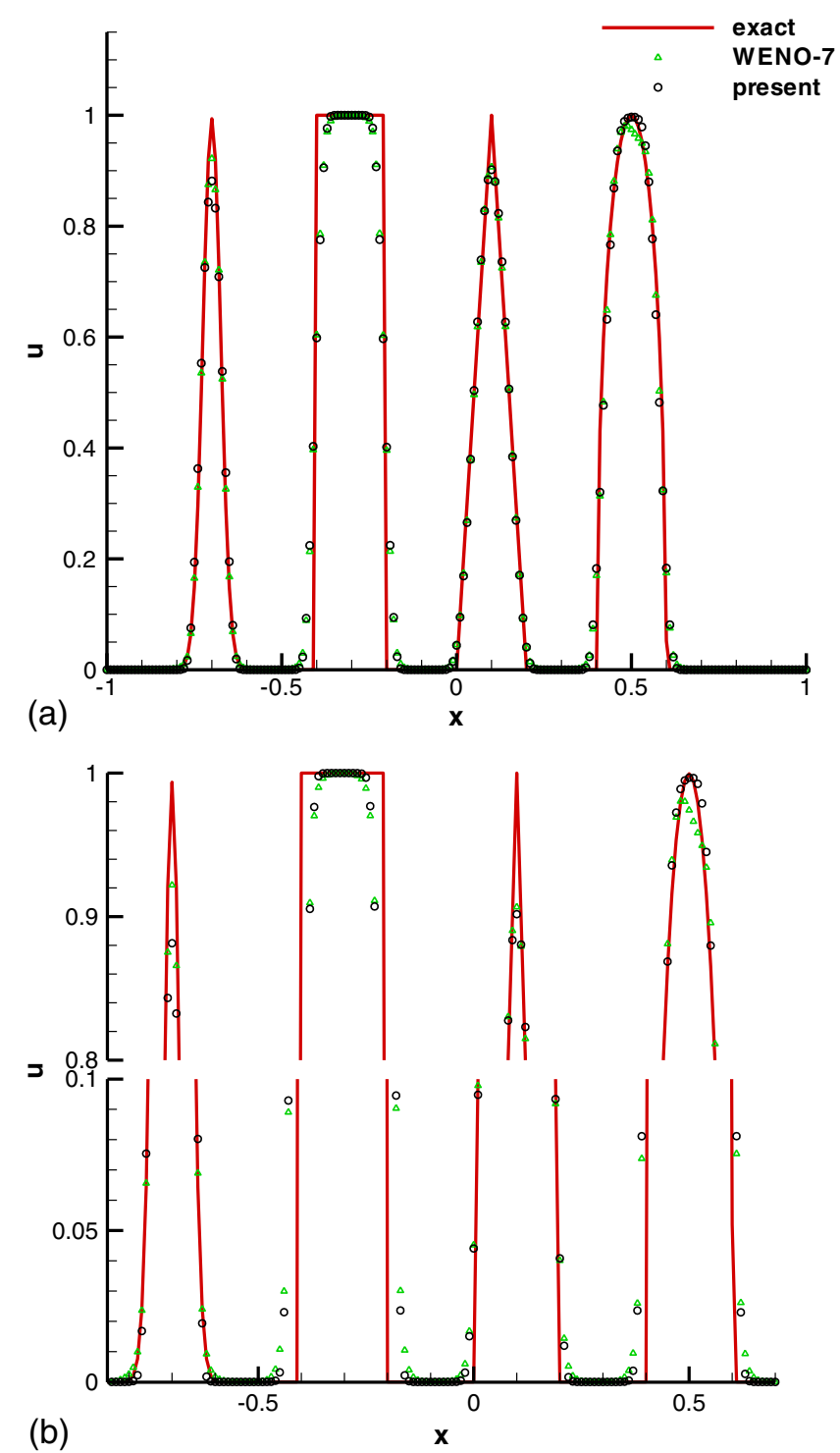

Figure 5. (a) Numerical results with the initial solution Equation (20), $t=6$. (b) Locally enlarged plot of Figure 5(a).

\subsection{One-dimensional shock tube problems}

The one-dimensional Euler equations of gas dynamics is solved. The first-order global LaxFriedrichs flux $[5,6,14]$ is used as the low-order building block for the high-order reconstruction of various WENO schemes.

One-dimensional Euler equations are written as

$$
\frac{\partial \mathbf{U}}{\partial t}+\frac{\partial \mathbf{F}}{\partial x}=0
$$

where $\mathbf{U}=\left[\begin{array}{l}\rho \\ \rho u \\ \rho e\end{array}\right], \mathbf{F}=\left[\begin{array}{l}\rho u \\ \rho u^{2}+p \\ (\rho e+p) u\end{array}\right], p=(\gamma-1)\left(\rho e-\rho u^{2} / 2\right), \gamma=1.4$.

1. Sod problem 


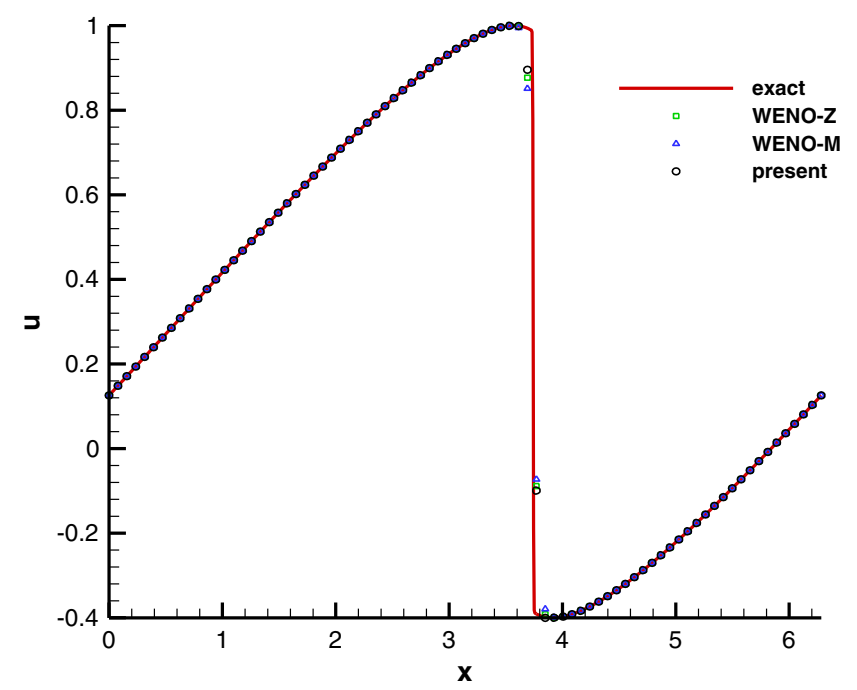

Figure 6. Numerical results of nonlinear transport equation $(21), t=2$.

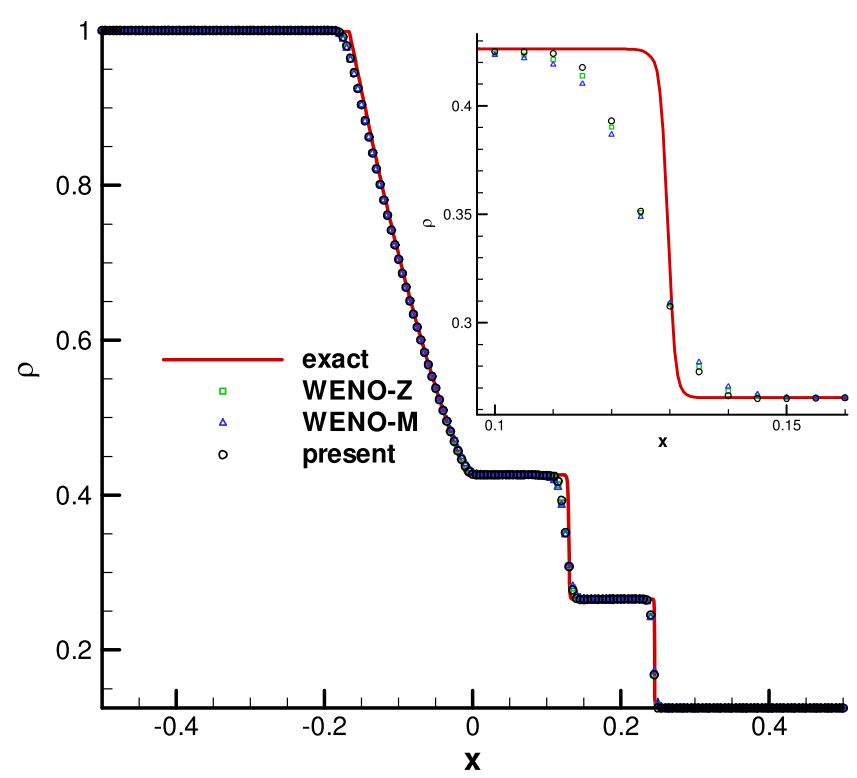

Figure 7. Density, Sod problem.

The initial conditions are

$$
(\rho, u, p)= \begin{cases}(1,0,1), & x<0 \\ (0.125,0,0.1), & x \geqslant 0\end{cases}
$$

The solution with $\mathrm{N}=200$ at $t=0.14$ is given in Figure 7. It can be seen that, near shocks, the present method is more accurate than both WENO-Z and WENO-M schemes.

2. Lax problem

The initial conditions are

$$
(\rho, u, p)=\left\{\begin{array}{l}
(0.445,0.698,0.3528), x<0 \\
(0.500,0.000,0.5710), x \geqslant 0
\end{array}\right.
$$

The solution with $\mathrm{N}=200$ at $t=1.3$ is given in Figure 8 . As in the sod problem, the new scheme is more accurate than the other fifth-order WENO schemes near shock waves. 


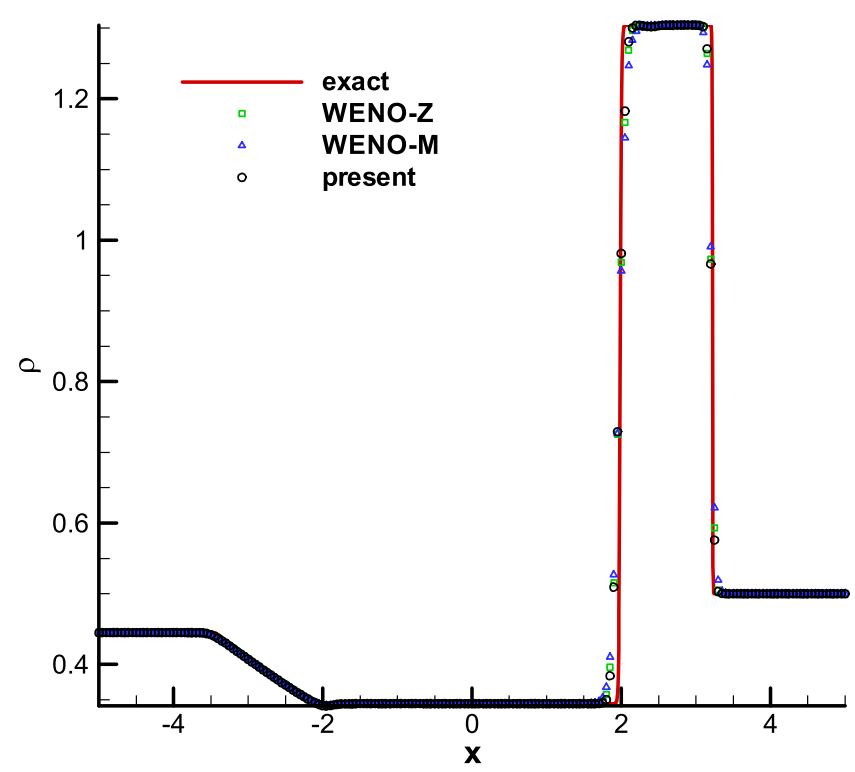

Figure 8. Density, Lax problem.

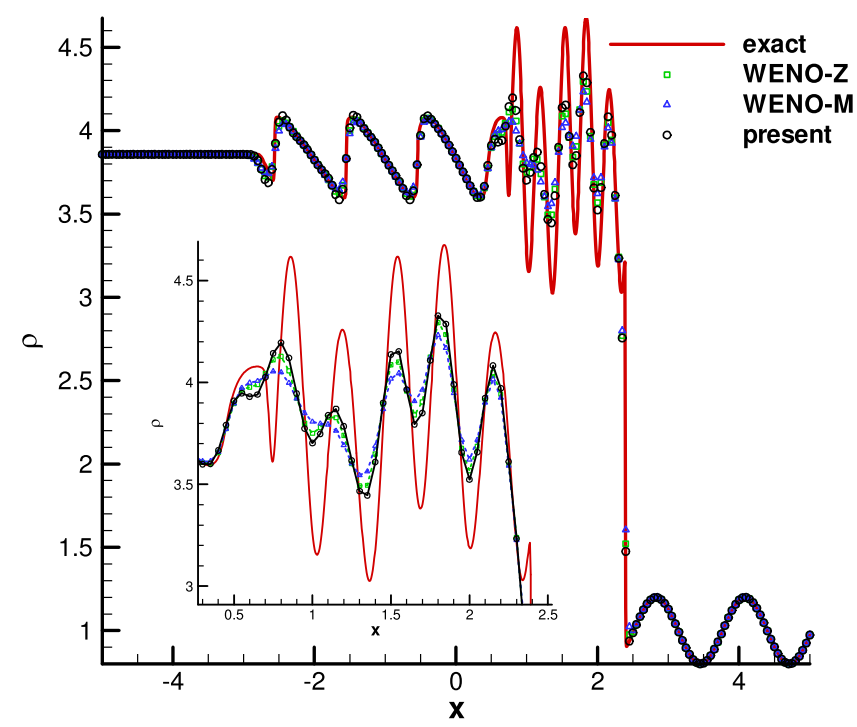

Figure 9. Density, Shu-Osher problem.

\section{Shu-Osher problem}

The initial conditions are

$$
(\rho, u, p)= \begin{cases}(3.857143,2.629369,10.33333), & \text { when } x<-4 \\ (1+\varepsilon \sin 5 x, 0,1), & \text { when } x \geqslant-4\end{cases}
$$

This case [15] represents a Mach 3 shock wave interacting with a sine entropy wave. The results with $\mathrm{N}=200$ at time $t=1.8$ are plotted in Figure 9. The 'exact' solutions are the numerical solutions of WENO-Z scheme with grid points of $N=2000$. It can be seen that, even in the smooth region, the present scheme are more accurate than WENO-Z and WENO$\mathrm{M}$ schemes. This indicates that, if the solution varies dramatically, the new method is less dissipative than the other two schemes. 
Figure 10 gives the results of the present method with three different meshes of $\mathrm{N}=200$, 400 , and 800 . It can be seen that the results with $\mathrm{N}=800$ agree well with the 'exact' solutions. It is also shown that the new method has good convergence behavior for complex problems.

4. Interacting blast waves

The initial conditions are

$$
(\rho, u, p)= \begin{cases}(1,0,1000), & 0 \leqslant x<0.1 \\ (1,0,0.01), & 0.1 \leqslant x<0.9 \\ (1,0,100), & 0.9 \leqslant x \leqslant 1.0\end{cases}
$$

The solution with $\mathrm{N}=400$ at $t=0.038$ is plotted in Figure 11. The comparison demonstrates that the present scheme can capture strong shock structures very well and is better than the other schemes.

\subsection{Two-dimensional advection equation}

The two-dimensional advection equation

$$
\left\{\begin{array}{l}
\frac{\partial u}{\partial t}+\frac{\partial u}{\partial x}+\frac{\partial u}{\partial y}=0, \quad-1 \leqslant x, y \leqslant 1 \\
u(x, y, 0)=u_{0}(x, y)
\end{array}\right.
$$

is used to test the accuracy of the schemes in the 2D case. The equation (23) is semi-discretized as

$$
\frac{d u_{i, j}(t)}{d t}=-\frac{1}{\Delta x}\left(h_{i+1 / 2, j}-h_{i-1 / 2, j}\right)-\frac{1}{\Delta y}\left(h_{i, j+1 / 2}-h_{i, j-1 / 2}\right)
$$

where the numerical fluxes $h_{i+1 / 2, j}$ and $h_{i, j+1 / 2}$ are constructed by the 1-D methodology along the grid lines of $x$-wise and $y$-wise, respectively. The initial condition is $u_{0}(x, y)=\sin (\pi(x+y))$, and the periodic boundary conditions in both $x$-wise and $y$-wise are applied. Table II shows the convergence rate of different schemes. For the smooth solution, three schemes achieve the fifth-order accuracy as similar as in 1-D case.

\subsection{Two-dimensional linear conservation law with variable coefficients}

The two-dimensional linear conservation law with variable coefficients

$$
\frac{\partial u}{\partial t}+\frac{\partial(-y u)}{\partial x}+\frac{\partial(x u)}{\partial y}=0, \quad-1 \leqslant x, y \leqslant 1
$$

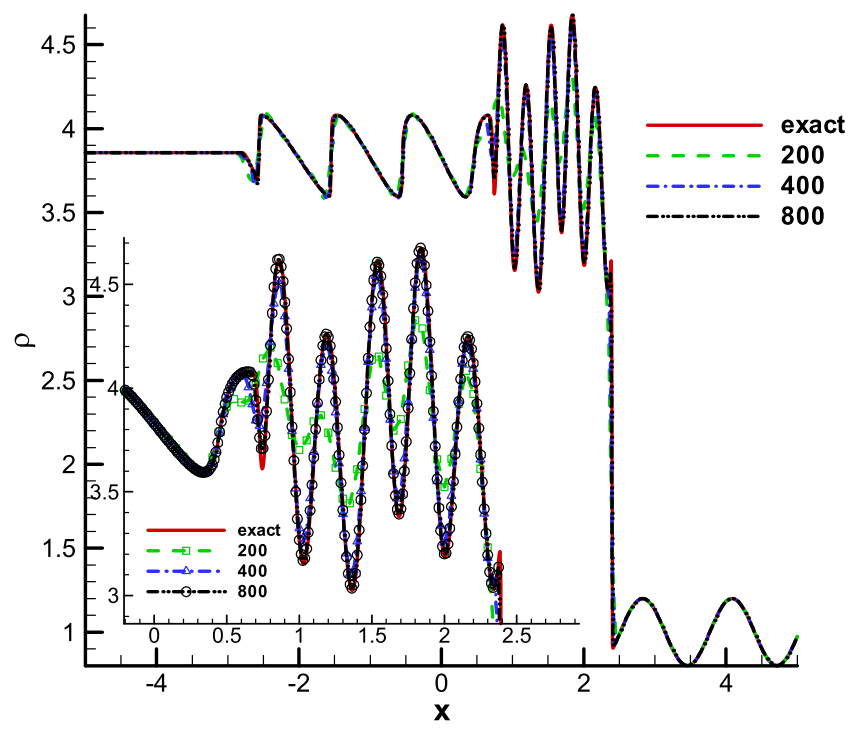

Figure 10. Density, present method with different meshes, Shu-Osher problem. 


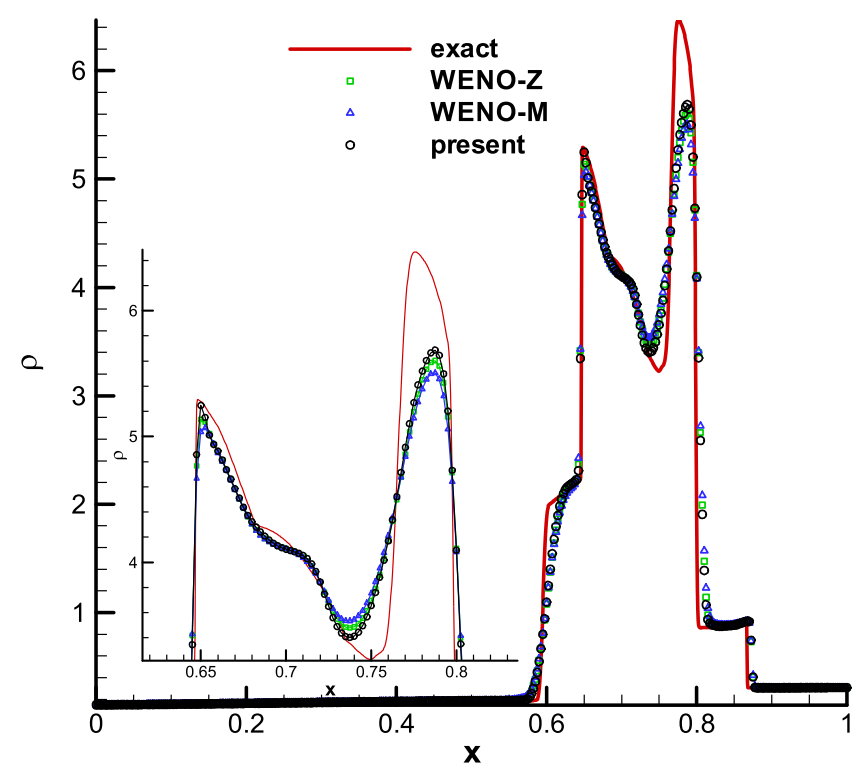

Figure 11. Density, interacting blast waves.

Table II. The accuracy in 2-D case, $\mathrm{T}=2$.

\begin{tabular}{cccccc}
\hline Scheme & $N_{x} \times N_{y}$ & $L_{1}$ error & $L_{1}$ order & $L_{\infty}$ error & $L_{\infty}$ order \\
\hline WENO-Z & $20 \times 20$ & $0.342283 \mathrm{E}-03$ & - & $0.214133 \mathrm{E}-03$ & - \\
& $40 \times 40$ & $0.102111 \mathrm{E}-04$ & 5.067 & $0.637584 \mathrm{E}-05$ & 5.746 \\
& $80 \times 80$ & $0.314224 \mathrm{E}-06$ & 5.022 & $0.199111 \mathrm{E}-06$ & 5.680 \\
& $160 \times 160$ & $0.979186 \mathrm{E}-08$ & 5.004 & $0.622505 \mathrm{E}-08$ & 5.658 \\
WENO-M & $320 \times 320$ & $0.305789 \mathrm{E}-09$ & 5.001 & $0.194579 \mathrm{E}-09$ & 5.653 \\
& $20 \times 20$ & $0.314390 \mathrm{E}-03$ & - & $0.210032 \mathrm{E}-03$ & - \\
& $80 \times 40$ & $0.995888 \mathrm{E}-05$ & 4.980 & $0.636855 \mathrm{E}-05$ & 5.625 \\
& $160 \times 160$ & $0.312491 \mathrm{E}-06$ & 4.994 & $0.199092 \mathrm{E}-06$ & 5.644 \\
present & $20 \times 20$ & $0.977631 \mathrm{E}-08$ & 4.998 & $0.622500 \mathrm{E}-08$ & 5.650 \\
& $40 \times 40$ & $0.995287 \mathrm{E}-05$ & 4.973 & $0.635348 \mathrm{E}-05$ & 4.985 \\
& $80 \times 80$ & $0.312471 \mathrm{E}-06$ & 4.993 & $0.199062 \mathrm{E}-06$ & 5644 \\
& $160 \times 160$ & $0.977603 \mathrm{E}-08$ & 4.998 & $0.622479 \mathrm{E}-08$ & 5650 \\
& $320 \times 320$ & $0.305600 \mathrm{E}-09$ & 5.000 & $0.194560 \mathrm{E}-09$ & 5.651 \\
\hline
\end{tabular}

with periodic boundary conditions is solved. The initial condition is chosen as the characteristic function of a circle with radius 0.5 . The problem represents a solid body rotation and is used to investigate the grid orientation effect as in Refs.[16, 17]. The results of the present method at $t=2$ with a mesh of $200 \times 200$ points are shown in Figure 12(a). It can be seen that the orientation effect is not strong. Figure 12(b) gives the comparisons along lines of $x=-0.02,-0.48$ and -0.52 ; it can be seen that the present method is the best one among the tested three schemes.

\subsection{Two-dimensional shock vortex interaction}

The two-dimensional governing equations can be written as

$$
\frac{\partial \mathbf{U}}{\partial t}+\frac{\partial \mathbf{F}}{\partial x}+\frac{\partial \mathbf{G}}{\partial y}=\frac{\delta}{\operatorname{Re}}\left(\frac{\partial \mathbf{F}_{v}}{\partial x}+\frac{\partial \mathbf{G}_{v}}{\partial y}\right)
$$




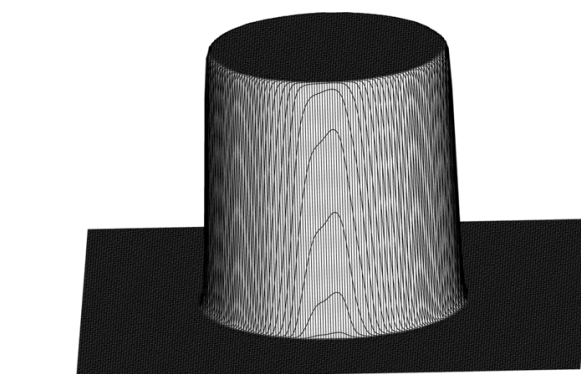

(a)
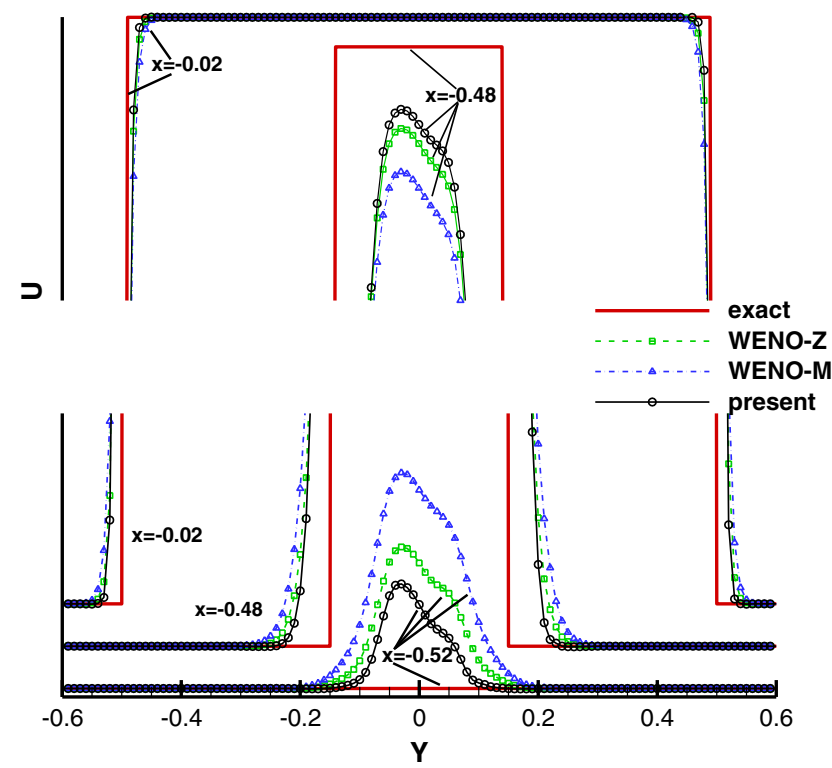

(b)

Figure 12. (a) Numerical results of Equation (25), present scheme. (b) Comparisons along lines of $x=$ $-0.02,-0.48$, and -0.52 , Equation (25).

where $\mathbf{U}=\left[\begin{array}{l}\rho \\ \rho u \\ \rho v \\ \rho e\end{array}\right], \quad \mathbf{F}=\left[\begin{array}{l}\rho u \\ \rho u^{2}+p \\ \rho u v \\ (\rho e+p) u\end{array}\right], \quad \mathbf{G}=\left[\begin{array}{l}\rho v \\ \rho u v \\ \rho v^{2}+p \\ (\rho e+p) v\end{array}\right], \quad \mathbf{F}_{v}=\left[\begin{array}{l}0 \\ \tau_{x x} \\ \tau_{x y} \\ u \tau_{x x}+v \tau_{x y}+q_{x}\end{array}\right]$,

$\mathbf{G}_{v}=\left[\begin{array}{l}0 \\ \tau_{x y} \\ \tau_{y y} \\ u \tau_{x y}+v \tau_{y y}+q_{y}\end{array}\right], \quad \tau_{x x}=\frac{2}{3} \mu\left(2 u_{x}-v_{y}\right), \quad \tau_{x y}=\mu\left(u_{y}+v_{x}\right), \quad \tau_{y y}=\frac{2}{3} \mu\left(2 v_{y}-u_{x}\right)$,

$q_{x}=\mu T_{x} /\left[(\gamma-1) M^{2} \mathrm{Pr}\right], \quad q_{y}=\mu T_{y} /\left[(\gamma-1) M^{2} \mathrm{Pr}\right], \quad p=(\gamma-1)\left(\rho e-\rho u^{2} / 2\right), \quad \gamma=1.4$. If $\delta=0$, Equation (26) is the two-dimensional Euler equations; If $\delta=1$, Equation (26) is the two-dimensional Navier-Stokes equations.

A two-dimensional shock vortex interaction problem taken from Ref. [3] is solved by using Euler equations (26). This problem describes the interaction between a stationary shock and a vortex. The computational domain is taken to be $[0,2] \times[0,1]$. A stationary Mach 1.1 shock is positioned at $x=0.5$ and normal to the $x$-axis. Its left state is $(\rho, u, v, p)=(1,1.1 \sqrt{\gamma}, 0,1)$. A small vortex is superimposed to the flow on the left of the shock and is centered at $\left(x_{c}, y_{c}\right)=(0.25,0.5)$. The vortex is described as a perturbation to the velocity $(u, v)$, temperature $(T=p / \rho)$, and entropy $\left(S=\ln \left(p / \rho^{\gamma}\right)\right)$ of the mean flow and denoted by the tilde values: 


$$
\left\{\begin{array}{l}
\tilde{u}=\varepsilon \tau e^{\alpha\left(1-\tau^{2}\right)} \sin \theta \\
\tilde{v}=-\varepsilon \tau e^{\alpha\left(1-\tau^{2}\right)} \cos \theta \\
\tilde{T}=-\frac{(\gamma-1) \varepsilon^{2} e^{2 \alpha\left(1-\tau^{2}\right)}}{4 \alpha \gamma} \\
\tilde{S}=0
\end{array}\right.
$$

where $\tau=r / r_{c}$ and $r=\sqrt{\left(x-x_{c}\right)^{2}+\left(y-y_{c}\right)^{2}}, \varepsilon$ indicates the strength of the vortex, $\alpha$ controls the decay rate of the vortex, and $r_{c}$ is the critical radius for which the vortex has the maximum strength. As in Refs. [3, 12], $\varepsilon=0.3, r_{c}=0.05$, and $\alpha=0.204$ are adopted in this paper.

The time step is taken as follows [18]

$$
\Delta t=\sigma \frac{\Delta t_{x} \Delta t_{y}}{\Delta t_{x}+\Delta t_{y}}, \text { with } \Delta t_{x}=\frac{\Delta x}{\max _{i, j}\left(\left|u_{i, j}\right|+c_{i, j}\right)}, \Delta t_{y}=\frac{\Delta y}{\max _{i, j}\left(\left|v_{i, j}\right|+c_{i, j}\right)}
$$

where $c$ is the speed of sound and $\sigma=0.5$ is the Courant-Friedrichs-Lewy number.

Figure 13 is the pressure contours at $t=0.60$ calculated by the present scheme. Figure 14(a)(c) is the comparison of the pressure along the center line of $y=0.5$. In order to show the accuracy of the new scheme, the results obtained by the WENO-Z scheme with a refined mesh of $2001 \times 401$ is given as the 'exact' solution. The results with the coarse mesh of $251 \times 101$ are compared. It can be seen that, behind the shock wave, the new scheme obtains almost the same maximum as the exact solution, and in the valley region, the present scheme is also more accurate than the other two WENO schemes.

Figure 15 shows the results of the present method with three different meshes of $251 \times 101,501 \times$ 201 , and $1001 \times 401$. It can be seen that, with refined meshes, the solutions converge to the 'exact' solution very well. Meanwhile, little difference between the maximal pressures calculated with different meshes shows that the present scheme is less dissipative even when the coarse meshes are used.

\subsection{Shock/shear layer interaction}

A shock/shear layer interaction problem from [19] is calculated to further demonstrate the low dissipation of the new scheme. The two-dimensional Navier-Stokes equations (26) are solved for this problem.

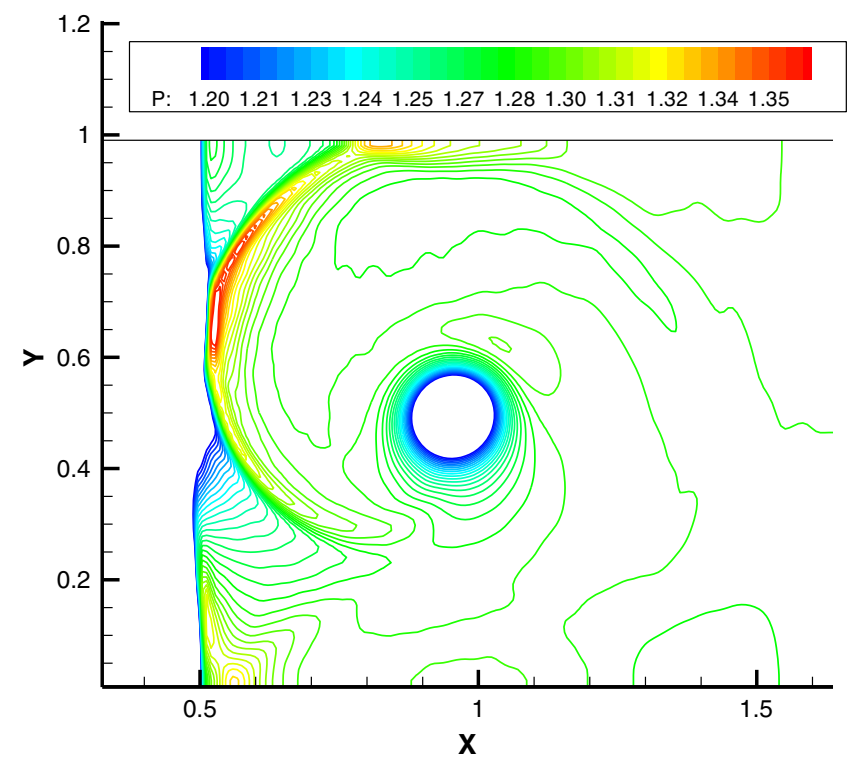

Figure 13. The pressure contours of present scheme, shock/vortex interaction problem. 

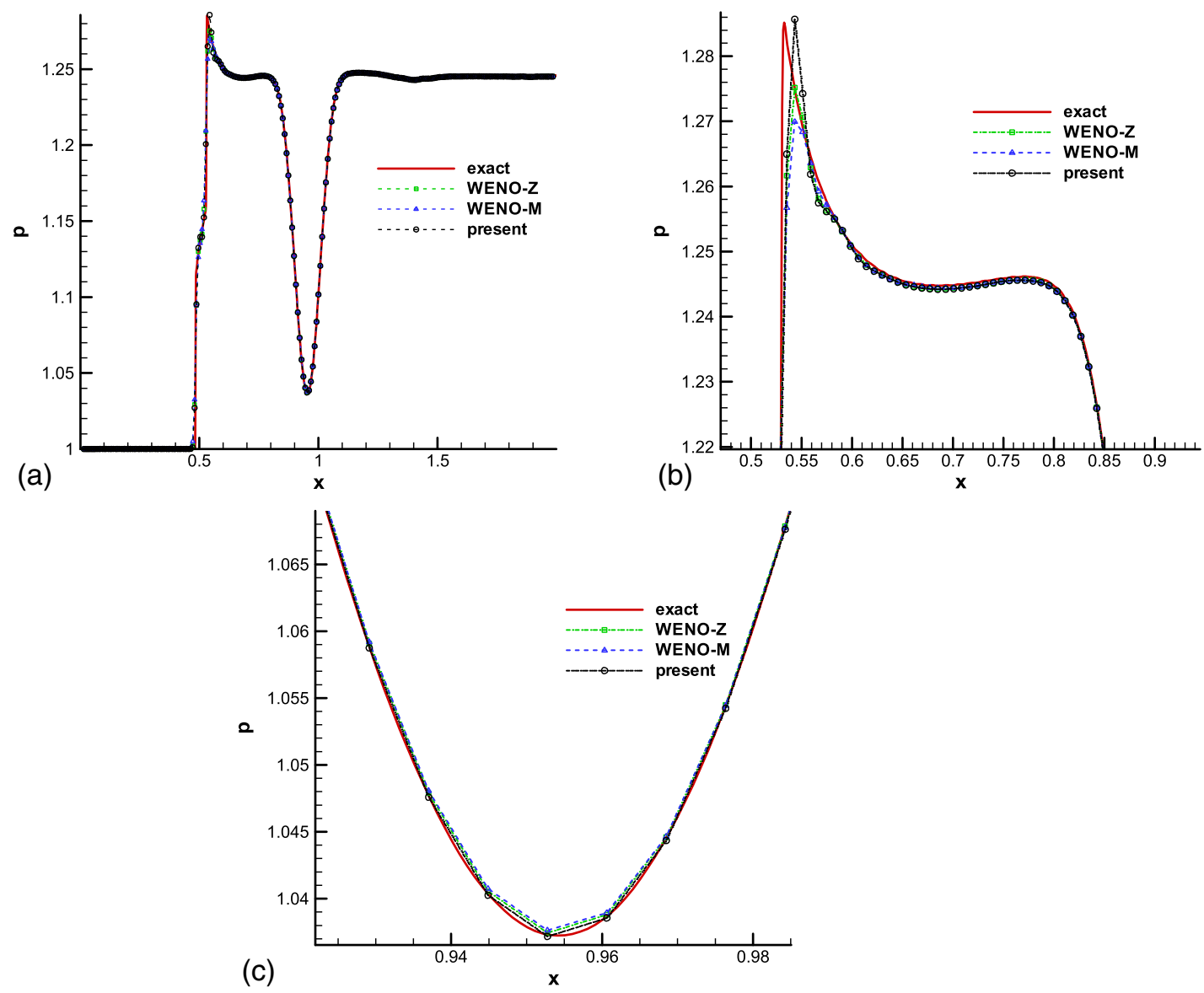

Figure 14. (a) The pressure distribution along the center line of $y=0.5$, (b) locally enlarged plot of Figure 14(a), and (c) locally enlarged plot of Figure 14(a).

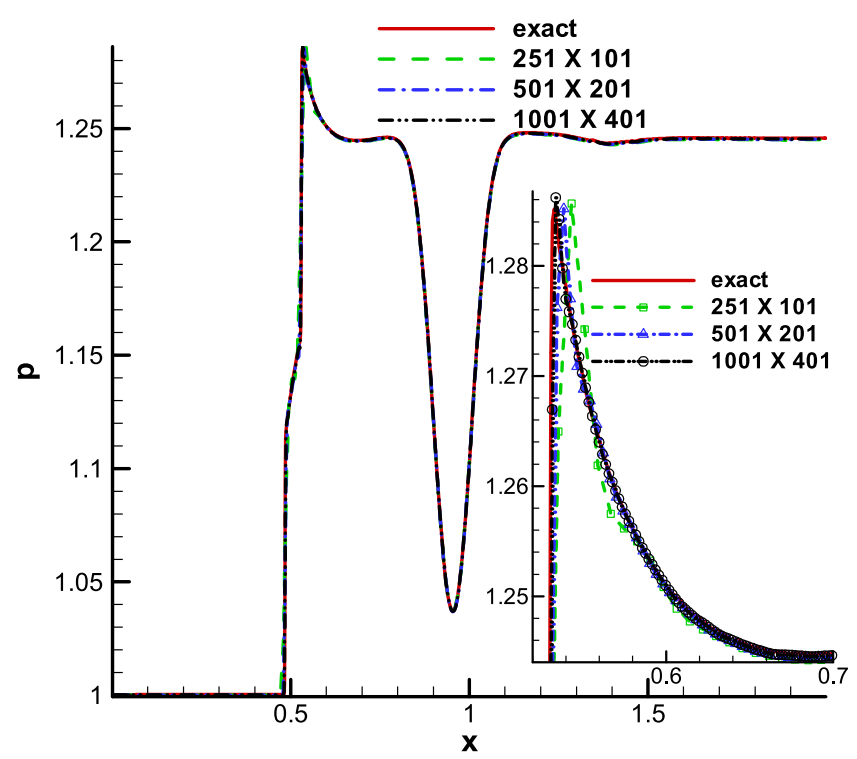

Figure 15. The pressure distribution along the center line of $y=0.5$, present method. 


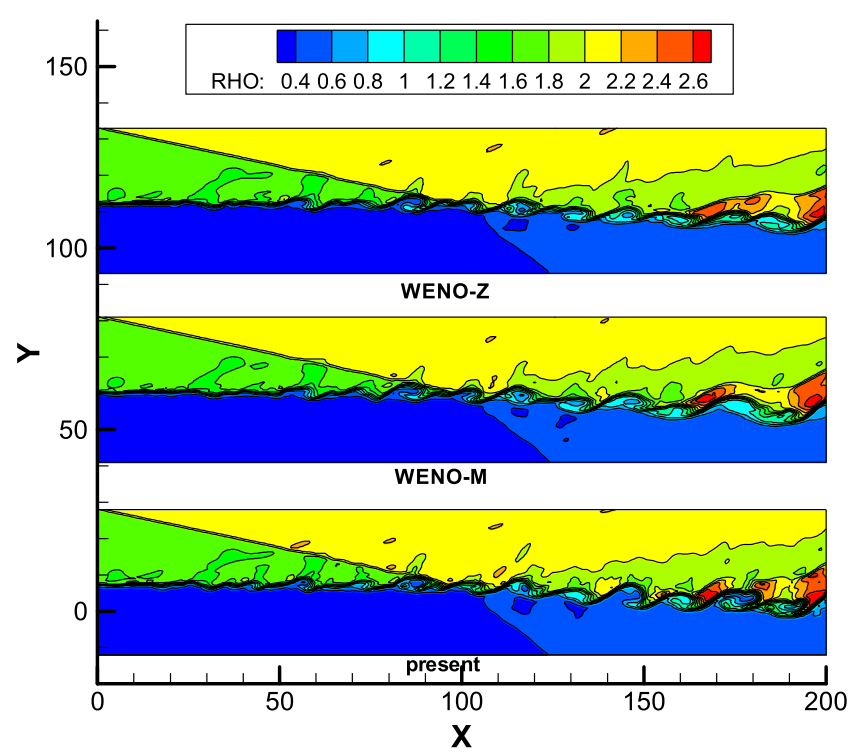

Figure 16. The density contours, shock shear layer interaction problem, $t=120$.

In this problem, the vortices arising from shear layer instability are forced to pass through a shock wave. An oblique shock with angle $\theta=12^{\circ}$ is made to impact on a spatially developing mixing layer at an initial convective Mach number of 0.5 . The computation domain is taken to be $[x, y]=[0,200] \times[-20,20]$. The inflow is specified with a hyperbolic tangent profile,

$$
u=2.5+0.5 \tanh (2 y)
$$

For the upper stream inflow, $\rho_{u}=1.6374, p_{u}=0.3327$; for the lower stream inflow, $\rho_{l}=$ $0.3626, p_{l}=0.3327$. The upper boundary condition is taken from the flow properties behind the oblique shock. The lower wall uses a slip condition.

Fluctuations are added to the v-component of velocity of the inflow as

$$
v^{\prime}=\sum_{k=1}^{2} a_{k} \cos \left(2 \pi k t / T+\phi_{k}\right) \exp \left(-y^{2} / b\right)
$$

with period $T=\lambda / u_{c}$, wavelength $\lambda=30$, convective velocity $u_{c}=2.68, b=10, a_{1}=a_{2}=$ $0.05, \phi_{1}=0$ and $\phi_{2}=\pi / 2$. The Prandtl number Pr is set to 0.72 , and the Reynolds number Re is chosen to be 500 .

The fourth-order central difference scheme [20] is used for the viscous terms. The uniform grids with the same grid number of $321 \times 81$ in [19] are used. The time step is also determined by Equation (27). The density contours are shown in Figure 16. It can be seen that three tested schemes can resolve the shock wave very well. But for the vortices, especially for the last four ones, the present scheme captures more clear vortex structures than the other two schemes.

\section{CONCLUSION}

By combining Henrick's mapping function and the idea of improving the accuracy of WENO-Z scheme one-by-one order, a new method for constructing WENO scheme is developed. In each step of weighting process, the sufficient condition for fifth-order convergence is kept, and hence, the final scheme can obtain fifth-order accuracy in smooth regions even containing critical points. The distinctive advantage of the new scheme is that it improves the accuracy of WENO scheme at transition points, and hence, its numerical dissipation near discontinuities is smaller than other fifth-order WENO schemes. Numerical examples show that the new scheme is accurate and robust. 


\section{ACKNOWLEDGEMENT}

This research work was supported by the National Natural Science Foundation of China under Grant 11272325.

\section{REFERENCES}

1. Liu XD, Osher S, Chan T. Weighted essentially non-oscillatory schemes. Journal of Computational Physics 1994; 115:200-212.

2. Harten A, Enquist B, Osher S, Chakravarthy S. Uniformly high order accurate essentially non-oscillatory schemes III. Journal of Computational Physics 1987; 71:231-303.

3. Jiang GS, Shu CW. Efficient implementation of weighted ENO schemes. Journal of Computational Physics 1996; 126:202-228.

4. Henrick AK, Aslam TD, Powers JM. Mapped weighted essentially non-oscillatory schemes: achieving optimal order near critical points. Journal of Computational Physics 2005; 207:542-567.

5. Borges R, Carmona M, Costa B, Don WS. An improved weighted essentially non-oscillatory scheme for hyperbolic conservation laws. Journal of Computational Physics 2008; 227:3191-3211.

6. Balsara DS, Shu CW. Monotonicity preserving weighted essentially non-oscillatory schemes with increasingly high order of accuracy. Journal of Computational Physics 2000; 160:405-452.

7. Gerolymos GA, Schal D, Vallet I. Very high order WENO schemes. Journal of Computational Physics 2009; 228:8481-8524.

8. Wang ZJ, Chen RF. Optimized weighted essentially non-oscillatory schemes for linear waves with discontinuity. Journal of Computational Physics 2001; 174:381-404.

9. Martin MP, Taylor EM, Wu M, Weirs VG. A bandwidth-optimized WENO scheme for effective direct numerical simulation of compressible turbulence. Journal of Computational Physics 2006; 220:270-289.

10. Shen YQ, Zha GC. Improvement of weighted essentially non-oscillatory schemes near discontinuities. 19th AIAA Computational Fluid Dynamics, 22 - 25 June 2009, San Antonio, Texas. AIAA, 2009; 2009-3655.

11. Shen YQ, Wang RQ, Liao HZ. A fifth-order accurate weighted ENN difference scheme and its applications. Journal of Computational Mathematics 2001; 19:531-538.

12. Shen YQ, Zha GC. Generalized finite compact difference scheme for shock/complex flowfield interaction. Journal of Computational Physics 2011; 23:4419-4436.

13. Shu CW, Osher O. Efficient implementation of essentially non-oscillatory shock capturing schemes. Journal of Computational Physics 1988; 77:439-471.

14. Ren YX, Liu ME, Zhang HX. A characteristic-wise hybrid compact-WENO scheme for solving hyperbolic conservation laws. Journal of Computational Physics 2003; 192:365-386.

15. Shu CW, Osher O. Efficient implementation of essentially non-oscillatory shock capturing schemes, II. Journal of Computational Physics 1989; 83:32-78.

16. Cockburn B, Shu CW. Nonlinearly stable compact schemes for shock calculations. SIAM Journal on Numerical Analysis 1994; 31:607-627.

17. Shen YQ, Yang GW, Gao Z. High-resolution finite compact difference schemes for hyperbolic conservation laws. Journal of Computational Physics 2006; 216:114-137.

18. Pirozzoli S. Conservative hybrid compact-WENO schemes for shock-turbulence interaction. Journal of Computational Physics 2002; 178:81-117.

19. Yee HC, Sandham ND, Djomehri MJ. Low-dissipative high-order shock-capturing methods using characteristicbased filters. Journal of Computational Physics 1999; 150:199-238.

20. Shen Y-Q, Zha G-Z, Chen X-Y. High order conservative differencing for viscous terms and the application to vortexinduced vibration flows. Journal of Computational Physics 2009; 228:8283-8300. 